\title{
Epigenetic Regulation of Toll-Like Receptor Signaling: Implications for Cancer Development
}

\author{
Shannon K. Boi Sherine F. Elsawa \\ Department of Biological Sciences, Northern Illinois University, DeKalb, Ill., USA
}

\section{Key Words}

Epigenetic mechanism · Toll-like receptor signaling · Cancer development

\begin{abstract}
The innate immune response acts in a myriad of ways to protect the host from pathogenic agents. This interplay requires changes in gene expression patterns that allow the host to effectively respond. Epigenetic mechanisms are important in this scenario. Understanding these mechanisms is of great interest for the development of appropriate therapies modulating the immune response. One of the important receptor families responsible for the activity of the innate immune system is the Toll-like receptor (TLR) family. This family is important for the innate immune response to infections and has been implicated to play a role in cancer. Understanding the epigenetic mechanisms regulated by TLR signaling is important for the development of novel therapies for a multitude of diseases including cancer. In this article, we present an overview of this pathway and its role in carcinogenesis, and we discuss the epigenetic mechanisms regulating its activity.

(C) 2013 S. Karger AG, Basel
\end{abstract}

\section{Toll-Like Receptor Signaling: Role in Innate Immune Response}

Initially identified in Drosophila, the Toll receptor was first noted in the spatial organization of dorsal-ventral patterns during embryogenesis [1] and was later discovered to be involved in the innate immune response to fungal infections in adult Drosophila bearing deficiencies in the receptor [2]. In the investigations that followed, there was a discovery and subsequent cloning of a human homolog of the Toll receptor - a Toll-like receptor (TLR) which shared similar characteristics and signaling components with the Drosophila Toll as well as with the cytoplasmic domain of the interleukin-1 receptor (IL-1R). This suggests that the immune response activated by Toll receptors and TLRs represented an ancient host 
Boi and Elsawa: Epigenetic Regulation of Toll-Like Receptor Signaling: Implications for Cancer Development

defense mechanism [3] and can also be found in evolutionarily distant organisms such as plants and puffer fish [4]. To date, there have been discoveries of 13 different TLRs between mice and humans with 10 functional TLRs in humans and 12 functional TLRs in mice [5, 6]. TLR1-TLR9 are conserved in both species, while TLR10 is not functional in mice due to a retroviral insertion [7], and TLR11-TLR13 have been lost from the human genome altogether $[5,6]$. Since these discoveries, the TLR family has become a pivotal point of investigation for not only the study of the innate immune system, but also for innate and adaptive cross talk, infectious diseases, acute and chronic inflammatory conditions as well as their role in proand antitumor consequences in cancer $[5,6,8]$. Naturally, TLRs have been found to be expressed in a number of malignancies and, therefore, their role in tumor progression has gained a central and crucial role in recent investigations. Such studies highlight the importance of TLRs as exploited processes in tumor biology that promote tumor growth by a number of mechanisms and aid in immune evasion, while other studies describe the antitumoral effects of TLRs. This leads to the understanding that TLRs can act as double-edged swords in the progression or treatment of cancer [6,9-12]. Antitumor therapeutics dates back over 100 years when Dr. William Coley demonstrated that repeated administrations of crude microbial extracts from Streptococcus pneumoniae and Serratia marcescens (Coley's toxin) promoted antitumoral responses in a variety of human cancers - a response that was undoubtedly attributed to the activation of TLRs to promote tumoricidal activity $[6,12]$. It is the accumulation of such evidence that pinpoints TLRs as viable therapeutic targets in a vast number of malignancies.

Biochemically, TLRs are type I transmembrane glycoproteins that are constituted by an extracellular domain marked by leucine-rich repeats which function primarily to recognize respective ligands and an intracellular domain that is marked by a Toll-like/IL-1R (TIR) that mediates downstream signaling. It has been demonstrated that in mice lacking each TLR, TLRs not only have distinct functions in terms of pathogen/danger (or damage)-associated molecular pattern (PAMP/DAMP) recognition, but also differences in immune responses [5]. As such, TLRs have been divided into subgroups based on the ligands that evoke their stimulation and their cellular localization. TLR1, TLR2, TLR4, TLR5, TLR6, and TLR11 are conventionally localized to the plasma membrane and primarily recognize microbial membrane components such as bacterial lipoproteins (TLR2), lipopolysaccharide (LPS) (TLR4), and flagellin (TLR5), while TLR3, TLR7, TLR8, TLR9, and TLR13 are conventionally localized to intracellular vesicles [endosomes, lysosomes, endoplasmic reticulum (ER), and endolysosomes] and primarily recognize microbial nucleic acids such as viral dsRNA (TLR3) [4, 5, 12-14]. Endogenous ligands, such as heat shock proteins, high-mobility group box 1, along with products of the extracellular matrix - such as fibronectin, biglycan, hyaluronan, and a variety of others - have also been shown to activate TLRs as well [12]. Among human TLRs, TLR10 is the only TLR that does not have a subgroup based on the established criteria and, as such, is currently an orphan receptor. This is partly due to the fact that murine TLR10 is not functional and, therefore, TLR10 mutant or genetically deficient mouse models have not been generated $[4,7]$. Trafficking of a majority of these receptors from the ER to their respective locations is mediated by ER-resident proteins such as PRAT4A and UNC93B1 [5, $14,15]$, while other resident proteins, such as gp 96, act as a general chaperone to aid in this orchestrated event [5]. Of note, TLR3, TLR7, and TLR9 need to be subjected to stepwise proteolytic cleavage in order to properly recognize respective ligands in the endolysosomes, while other TLRs, such as TLR4, may require the support of coreceptors in order to facilitate proper ligand binding $[15,16]$.

In order to mediate biological functions, TLRs form homo- and heterodimers with each other. Most TLRs form homodimers to transduce signals, with the exception of TLR2, which can heterodimerize with either TLR1 or TLR6 to recognize distinct peptides from different 
sources to mediate different biological effects [17]. TLR10 can also form homodimers with itself as well as heterodimerize with TLR1 and TLR2, but it has not been shown to interact with TLR6 [7]. After receptor engagement with ligand, there is an initial recruitment of cytosolic TIR-containing adaptor proteins such as myeloid differentiation primary response gene 88 (MyD88), TIR domain-containing adapter-inducing interferon- $\beta$ (TRIF/TICAM1), MyD88 adaptor-like (Mal or TIR domain-containing adaptor protein, TIRAP), TRIF-related adaptor molecule (TRAM/TICAM2), and sterile $\alpha$ - and armadillo-motif-containing protein (SARM) [6]. There are two subsequent pathways based on TIR adaptor utilization - an MyD88mediated pathway and a TRIF-mediated pathway.

\section{MyD88-Dependent Pathway}

MyD88 can associate with TIRAP to induce an MyD88-dependent pathway. Noncanonically, TRIF can associate with TRAM to induce MyD88-independent signaling, while SARM has been noted to be a negative regulator of TRIF [17-19]. Once MyD88 and TRAM have been recruited to the TIR domains of the TLR cytoplasmic region, this initiates signaling events through the recruitment of IL-1R-associated kinases (IRAKs) and tumor necrosis factor (TNF) receptor-associated factor 6 (TRAF6) which leads to the activation of further kinases and ultimately causes the activation of NF- $\kappa \beta, \mathrm{p} 38, \mathrm{JNK}$, and p42/p44 MAP kinases (fig. 1) $[19,20]$. The activation of this MyD88-dependent pathway leads to the formation of components that enhance immune responses such as the production of proinflammatory cytokines. Nearly all TLRs use this MyD88-dependent canonical pathway with the exception of TLR3, which uses the TRIF-dependent pathway $[14,20]$.

\section{TRIF-Dependent Pathway}

Alternatively, the TRIF-dependent pathway causes activation of TRAF3 and subsequent activation of interferon regulatory factor 3 (IRF3), which leads to the production and secretion of type I interferons (IFNs) such as IFN- $\beta$ (fig. 1) [6, 20]. TRIF-mediated induction of type I IFNs can also cause the upregulation of costimulatory molecules and MHC class II molecules by macrophages and dendritic cells (DCs) in a paracrine or autocrine fashion, which aids in instructing adaptive immune responses [21]. In addition, some proinflammatory responses can be activated in both a TRIF- and MyD88-mediated manner. For example, IRF7 acts as a transcriptional activator of type I IFNs and can be activated through both pathways [14] as can a number of other regulatory proteins such as mitogen-activated protein kinase (MAPK) effectors and NF- $\kappa \beta$ [22]. Furthermore, it has been shown that TLR4 can activate the noncanonical TRIF pathway via dynamin-dependent endocytosis and subsequent sequestering of the receptor in early endosomes. This allows for the recruitment of TRAM and subsequently TRIF to mediate the appropriate biological effects (fig. 1) [23].

As investigations continue to unravel additional details of the components that mediate TLR signaling, there have been a number of additional pathways that have been found to cross-talk with, or are mediated by, TLR signaling. One important example is the induction of apoptosis. It has been shown through multiple studies that TLRs can activate apoptotic cascades. TLR3 can activate apoptosis through both the intrinsic and extrinsic pathways through the binding of the respective synthetic ligand polyinosinic:polycytidylic acid [poly(I:C)] in endothelial cells [24]. TLR2 can activate apoptosis through MyD88 which in turn activates the Fas-associated death domain-caspase-8 pathway in monocytes/macrophages through direct death-domain interactions [25-27]. It has also been reported that Mycobacterium leprae can stimulate TLR2-activated apoptosis of Schwann cells to cause nerve damage, a clinical hallmark of leprosy [28], suggesting that the fundamental understanding of apoptotic induction by TLRs is of great clinical value. Similarly, Gram-negative bacteria, such as Yersinia enterocolitica and Escherichia coli, can induce apoptosis through 


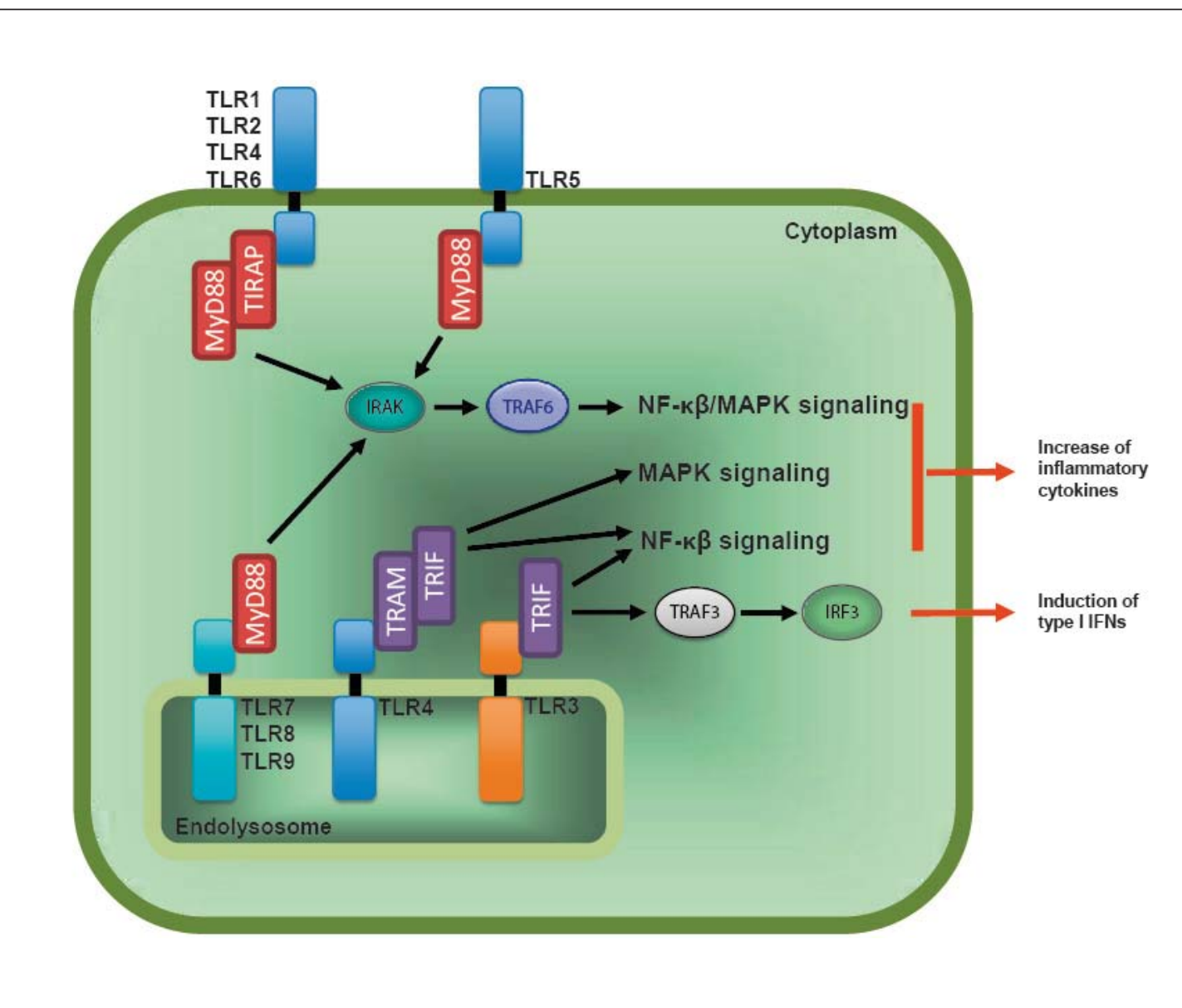

Fig. 1. Presentation of intracellular signaling events through MyD88- and TRIF-dependent pathways. Membrane (TLR1, TLR2, TLR4, TLR5, and TLR6) and endolysosomal (TLR3, TLR7, TLR8, and TLR9) signaling by TLRs is shown. MyD88-dependent signaling through the activation of IRAK and subsequently of TRAF6 (among other components) furthers the MAPK and NF- $\kappa \beta$ signaling to induce the production and secretion of inflammatory cytokines. TRIF-dependent signaling through activation of TRAF3 and subsequently of IRF3 (among other components) causes induction of type I IFNs and also stimulates NF- $\kappa \beta$ signaling. It is important to note that TLR4 can mediate biological effects through MyD88-dependent signaling on the plasma membrane and can also stimulate late MAPK and NF- $\kappa \beta$ signaling in endolysosomes through TRIF-dependent activation.

TLR4-mediated TRIF activation, which can then signal through receptor-interacting protein 1 to ultimately stimulate the Fas-associated death domain-caspase-8 pathway in macrophages and DCs [17, 29-31]. While this evidence is enough to suggest that pathogenic microbes may use TLRs as a way to destroy host immune defenses, activation of the apoptotic pathway initiated by TLRs fulfills multiple roles in the progression of the innate and adaptive immune responses to potential threats [25]. Furthermore, there has been additional evidence that TLRs can interact with other pathways such as the Notch signaling pathway [32-34], which is a pathway involved in cell fate decisions such as cell growth, differentiation, and survival during development of hematopoietic systems as well as various other systems [32,33]. These findings have spurred additional investigations into TLRNotch signaling where it has been found to exacerbate conditions such as calcific aortic stenosis [34]. 


\section{Toll-Like Receptor Signaling and Its Implications in Disease}

Overall, it is the activation of TLRs by their respective ligands that triggers a multifaceted response which then primes the stimulation of specific adaptive responses involving $\mathrm{T}$ and $\mathrm{B}$ lymphocytes [35] and provides the host with immediate inflammatory protective measures against the elicited threat. A role for inflammation in tumorigenesis is now generally accepted. This is marked by the understanding that an inflammatory microenvironment is an essential component of tumor cell biology [36]. Along with this inflammation, however, comes not only promotion of tumorigenesis, but also some marked responses that can be utilized to combat cancer with cancer immunotherapy and which show augmented responses to chemotherapy [36]. Nevertheless, it is with no surprise that TLRs can also be included in this classification. Uncontrolled TLR signaling can provide protumorigenic effects that allow the malignant cells to proliferate and evade immune responses, while other elicited responses can induce antitumoral immune responses to suppress tumor progression [6]. Below, we examine the role of TLRs in a variety of solid tumors and hematological malignancies.

\section{Breast Carcinoma}

While the chances of developing invasive breast cancer are a little less than $12 \%$, breast cancer still remains one of the most common cancers among American women to date [37]. One study conducted in 2010 analyzed the TLR expression of TLR3, TLR4, and TLR9 in breast cancer as well as its relation to distant metastasis [38]. It was found that TLR3 expression was associated with high probability of metastasis, while TLR4 expression by mononuclear inflammatory cells was also associated with an increased probability of metastasis [38]. Interestingly enough, fibroblast-like cell expression of TLR9, however, was associated with a low metastasis rate [38]. TLR4 was also identified as a promoter of metastasis through stimulation by LPS as well as molecules from damaged tumor cells (DTC-Ms) on the metastatic breast cancer cell line MDA-MB-231 [39]. This stimulation of TLR4 caused the upregulation of the $\alpha v \beta 3$ expression, a common characteristic of the metastatic phenotype in breast cancer. Alternatively, TLR4 stimulation caused the downregulation of TPM1 and maspin, both of which inhibit matrix proteolysis and mediate suppression of the invasive migration of tumor cells [39]. It has also been noted that polymorphisms in the Tlr4 gene can be a potential marker for early relapse in breast cancer patients. Patients harboring a loss-of-function Asp299Gly mutation have been found to relapse earlier upon anthracycline-based chemotherapy when compared to their functionally competent counterparts [40]. In addition, increased susceptibility to breast cancer development was also found in not only the Asp299Gly mutation, but also another polymorphism, a Thr299lle [40]. TLR4 was the highest expressed TLR in MDA-MB-231 breast cancer cells and when knocked down, the abrogation of TLR4 highly inhibited growth and proliferation of the cancerous cells as well as markedly depressed TLR4-related cytokines such as IL-6, IL-12, and nitric oxide. The latter are involved in attracting and activating inflammatory cells, which may underwrite the ability of the tumor cells to be resistant to immune cell attack and enable evasion from immune surveillance $[37,41]$.

The synthetic ligands poly(I:C) and poly(A:U) have been utilized as adjuvant therapies for a variety of cancers, including breast cancer, in an attempt to mount an innate immune response against cancerous cells. However, further investigations have shown that another attribution to this success might be the presence of a direct proapoptotic effect on these cancerous cells when stimulation of TLR3 occurs [42]. Indeed, multiple studies have shown that TLR3 stimulation directly inhibits the proliferation of tumor cells and induces apoptosis [6, 42-45]. In addition to TLR3 stimulation, retinoic acid (RA) can regulate cellular proliferation, differentiation, and apoptosis, and has been shown to inhibit and prevent breast cancer 
growth and carcinogenesis. Therefore, it has recently been investigated whether or not utilizing both RA inhibition as well as the apoptotic effect of TLR3 stimulation could work synergistically to improve apoptotic responses [46]. Interestingly enough, it appears as if the RA/poly(I:C) interaction favors an improved mechanism for apoptosis, one that induces upregulation of TLR3, along with other components by RA and subsequent downstream effects, as well as TLR3-mediated signaling that induces apoptosis through type I IFN autocrine signaling, caspase-3 and -8 activation as well as TNF-related apoptosis-inducing ligand signaling [46]. Overall, this proposed costimulatory therapy seems promising.

\section{GastrointestinaI Malignancies}

Hepatocellular carcinoma (HCC) is the third leading cause of death worldwide, and is regarded as one of the most aggressive human cancers due to the recurrence of metastasis [47]. A majority of HCC occurs in patients that have a history of chronic liver diseases, particularly with viral hepatitis as well as liver cirrhosis. These etiological agents promote a very inflammatory environment [48]. It is therefore understandable that the cross talk between the tumor cells and the surrounding inflammatory microenvironment is required for development and sustained progression of HCC. It is this microenvironment that plays key roles for many processes including tumor invasion, epithelial-mesenchymal transition, and metastasis [47]. LPS-induced TLR4 was found to activate NF- $\kappa \beta$ which caused an increase in Snail leading to upregulation of epithelial-mesenchymal transition as well as increased invasion of HCC cells [49]. Similarly, when TLR4 expression levels were measured in 106 HCC tissue samples, TLR4 expression seemed to be significantly higher in tissues with cirrhosis [49]. This high expression of TLR4 was noted in patients with poor cancer-free or overall survival compared to those with low expression of the receptor [49]. When MyD88 is highly expressed in HCC cells, it promotes tumor progression through TIR-independent activation of NF- $\kappa \beta$ by activating both the PI3K/AKT pathway as well as the p38/ERK pathway and possibly through antiapoptotic methods [50]. It is also of note that high expression of MyD88 caused enhanced cellular proliferation of tumor cells and, therefore, can be associated with poor prognosis of cancer-free and overall survival rates [50]. Poly(I:C) stimulation of TLR3, however, acts in an antitumorigenic fashion, both by slowing cellular proliferation of HCC cells and increasing apoptosis. Likewise, TLR3 stimulation also enhanced the proliferation of both T lymphocytes as well as NK cells which enhanced tumor-suppressive activities [48].

Pancreatic ductal adenocarcinoma is the fourth most common cause of cancer death in the United States, and has a 5-year survival rate of less than 6\% [51,52]. The tumor microenvironment plays a huge role in the promotion of pancreatic cancer [51]. As such, TLRs have played a critical role in understanding the interplay between the inflammatory environment and the malignancy. TLR7 was found to play a significant role in mediating pancreatic tumor progression by numerous ways. First, TLR7 was found to be highly expressed in both neoplastic ductal epithelial cells and inflammatory cells (roughly $50 \%$ of B cells, $65 \%$ of $\mathrm{T}$ cells, $40 \%$ of neutrophils and nearly all macrophages and DCs) within the tumor microenvironment compared to none or minimal expression of TLR7 in normal pancreata, respectively [51]. Furthermore, ligation of TLR7 with ssRNA40 induced vigorous tumor growth in Krastransformed epithelial cells, TLR7 ligation induced a gain of p53, p21, p27, p-p27, retinoblastoma protein, SHPTP1 protein, and cyclin B1 and a loss of p16, PTEN, and cyclin D1. In correlation with the loss of PTEN, an increase in pAkt and TGF- $\beta$ was noted, and subsequent activation of the PI3K/AKT pathway resulted in increased intrapancreatic expression of STAT3 and p-STAT3 and their associated antiapoptotic genes c-Myc and Bcl-xL [51]. Remarkably, treatment with TLR7 inhibition prevented progression by downregulating expression of p21, p27, p-p27, cyclin B1, and p-STAT3 [51]. In addition, it was also found that TLR7 upregulated Notch expression, providing evidence that TLR-Notch interplay drove 
Notch signaling toward inflammatory end products and caused a self-amplifying loop that promoted inflammation through cytokine production [51, 53]. However, mice with inflammatory cells lacking TLR7 were exclusively protected from neoplasia, suggesting that TLR7 ligation drives cancer progression by influencing stromal inflammation [51, 53]. TLR2 has been considered as a pancreatic cell surface marker as it has been shown to be expressed in over $70 \%$ of pancreatic tumors but not in normal tissue [52, 54]. As such, TLR2 agonist ligands are being explored as potential targets for pancreatic cancer imaging or immune adjuvants for use in immunotherapies [52]. TLR4 has also been implicated in pancreatic tumor progression due to the fact that TLR4 is highly expressed on leukocytes within the pancreatic tumor microenvironment, where endogenous ligands can be readily found, and engagement of TLR4 results in accelerated carcinogenesis [55]. It was also noted that inhibition of TLR4 signaling via the TRIF pathway provides a protective role against tumor progression $[53,55]$. Inhibition of signaling via the MyD88 pathway, however, causes increased inflammation and subsequent malignant progression mediated by DCs, which promote TH2 polarization that serve as the effectors of the increased inflammation $[53,55]$.

\section{Lung Carcinoma}

Despite many advances in therapeutics for lung carcinoma, only $15 \%$ of patients survive more than 5 years from the time of diagnosis [56]. Due to this poor prognosis, lung carcinoma is considered one of the most common causes of death worldwide [56] leading researchers to study TLR signaling for new therapeutic options. TLR7 and TLR8 have been shown to be linked to inflammation, tumor growth, and chemoresistance in lung cancer cells [57]. This evidence is highlighted by the fact that TLR7 was expressed by bronchial cells, immune cells, and in tumor cells (in $~ 70 \%$ of patients), while TLR8 was primarily expressed only by bronchial and tumor cells, suggesting that adjuvant immunotherapies using agonists to these TLRs may also cause significant problems for patients [57]. TLR9 levels were found to be highly expressed in tumors, while TLR4 was found to be moderately expressed compared to the weak expression of both receptors in normal tissue [58]. Remarkably, while there was no correlation between TLR4 and TLR9 expression with patient age, gender and smoking history (among other clinicopathological features), there was a positive correlation between TLR4 and degree of differentiation of the tumor cell, suggesting that TLR4 and TLR9 may be involved in the development of lung cancer [58]. Given these high levels of TLR9 expression, TLR9 has been found to promote tumor progression by enhancing proliferation and invasive abilities of tumor cells when stimulated with CpG oligonucleotides, a widely used adjuvant in anticancer therapies [59,60]. Nickel has been shown to be an agonist for TLR4 [61], and in recent studies, nickel could also contribute to the progression of human lung cancer by increasing the metastatic potential of the tumor cells by elevating levels of IL-8, TGF- $\beta$, MMP2, and MMP9 by activation of the MyD88-dependent NF- $\kappa \beta$ and p38 MAPK pathways [62]. Moreover, the downregulation of TLR4 or MyD88 could significantly inhibit the invasive potential of the cells when exposed to nickel [62].

\section{Hematological Neoplasms}

Since it is largely accepted in the field that neoplastic B lymphocytes are, to a larger extent, thought to represent normal B cells blocked at different stages in development and differentiation, these neoplastic cells are used as models to study their wild-type counterparts [35] and the same can be applied to T lymphocytes. While understanding the normal physiology of TLR expression and subsequent biological functions mediated thereafter in lymphoid cells is fundamental to the core of basic science and immunology, these investigations have led to the discovery of multiple TLR signaling pathways that lymphomas utilize to further their own proliferation and disease in patients. An oncogenic MyD88 has been re- 
cently identified in the activated B cell-like subtype of diffuse large B cell lymphoma in which a gain-of-function mutation (L265P) in the TIR domain of MyD88 causes aberrant IRAK activation and subsequently promotes the survival of malignant cells through JAK-STAT and $\mathrm{NF}-\kappa \beta$ signaling $[63,64]$. It was also noted that this MyD88 somatic mutation was also identified in gastric mucosa-associated lymphoid tissue lymphomas [63] as well as in a majority of Waldenström's macroglobulinemia patients and in non-IgM lymphoplasmacytic lymphoma ( 90\% of tumor samples) $[65,66]$, suggesting that this mutation could possibly be therapeutically targeted in other lymphomas. Interestingly enough, targeting of this oncogenic MyD88 using RNA interference methods had little toxic effect on germinal center B cell-like diffuse large B cell lymphoma, Burkitt's lymphoma, mantle cell lymphoma, and multiple myeloma (MM) [63]. This could be attributed to the fact that other lymphomas utilize different driving forces of TLR signaling to further their progression. In the case of mantle cell lymphoma, stimulation of TLR4 by LPS promoted tumor growth and played an imparting role in the resistance of cytotoxic T lymphocyte-mediated destruction [67]. MMs also use TLR4 in malignant progression, promoting proliferation in part by the proliferative abilities mediated by IL- 6 production, preventing apoptosis and facilitating immune evasion [68-70]. To some extent, TLR9 may play a role in such progression as well [68].

\section{Toll-Like Receptors and the Governing Epigenetic Mechanisms: Roles in Cancer Progression}

Epigenetic regulation is the inherited variation that occurs without changes in the DNA sequence. It is this inherited variation that allows for the establishment of cell-specific expression programs by modifications to the chromatin template [71]. Chromatin organization is dependent on the structuring of nucleosomes - $147 \mathrm{bp}$ of DNA wrapped around an octamer of histone proteins - and is the basic repeating unit of chromatin [72, 73]. Nucleosomes are then subjected to further higher-order chromatin structures which serve as a sentinel to protect the genome from potential damage [74]. Furthermore, this causes difficulty in transcribing genes, therefore implicating chromatin structures to be the most basic regulatory mechanisms for gene expression in eukaryotes [74]. However, modifications to the chromatin template can be utilized to alter DNA accessibility to transcriptional machinery. Such modifications include histone posttranslational modifications (which can then be subsequently modified in a large variety of ways including acetylation, methylation, phosphorylation, ADP-ribosylation, ubiquitination, sumoylation, and biotinylation [71, 75-77]), nucleosome repositioning, and DNA methylation which help to drive changes in gene expression and ultimately confer specific biological responses [71, 76]. DNA methylation, especially at promoters and enhancers, is mainly coupled with transcriptional silencing, whereas histone modifications are involved in both positive and negative regulation of transcription $[77,78]$. The alternative roles of histone modifications can be seen through such examples as acetylation of histone 3 and 4 or di- or tri-methylation (me) of H3K4 are commonly referred to as activators of transcription, while modifications localized to inactive genes, such as $\mathrm{H} 3 \mathrm{~K} 9 \mathrm{me}$ and $\mathrm{H} 3 \mathrm{~K} 27 \mathrm{me}$, are often portrayed as more heterochromatin regulations [73]. It is not surprising that understanding epigenetic regulation is an emerging option in investigations and clinical therapies of malignancies. In this case, how epigenetic regulation is mediated by TLR signaling can further cancer progression, both in the cancer cells as well as in the microenvironment.

Tumor-associated macrophages are known to play crucial roles in solid tumors, which, among other functions, work to produce higher amounts of IL-10 - an immunosuppressive cytokine [22]. TLRs have been noted to play a role in the induction of IL-10. TLR stimulation 
caused ERK1/2 MAPK activation, which further triggered histone phosphorylation at the IL-10 promoter, causing enhanced IL-10 production [22]. TNF- $\alpha$ in the bone marrow microenvironment supports MM by activating NF- $\kappa \beta$, which provides pleiotropic effects to further malignancy $[79,80]$. TLR4 has been implicated in epigenetic regulation of the TNF- $\alpha$ promoter, and LPS stimulation increased the H3 and H4 acetylation of the TNF- $\alpha$ locus [81].

In the case of endemic Burkitt's lymphoma, TLR9 may play a role in the survival of the malignant B cells by preventing the lytic phase of the Epstein-Barr virus [82]. Activation of TLR9 causes decreased levels of phosphorylated histone $\mathrm{H} 3$ and acetylated histones $\mathrm{H} 4$ and $\mathrm{H} 3$ bound to the Epstein-Barr virus master regulatory lytic gene promoter, thus preventing the virus from entering the lytic phase [82].

Histone deacetylases (HDACs) are enzymes that remove acetyl groups from histones. They have been linked to the pathogenesis of diseases, including cancer, and are currently being explored as therapeutic agents in patients with hematological malignancies [83]. While the outlook is promising, HDAC inhibitors have a plethora of effects on the immune system, in particular on TLR signaling. LBH589, a novel pan-histone deacetylase, was found to repress a variety of cytokines including IL-6, IL-10, TNF- $\alpha$, IL-12, and IL-23 [84]. TSA, a prototypical broad-spectrum inhibitor of class I, II, and IV HDACs, inhibited LPS-induced upregulation of genes including TLRs, CD14, and MD-2 along with a variety of others [83]. Due to the fact that HDACs have adverse negative effects on the immune system, it has been suggested that the use of HDAC inhibitors as anticancer agents may increase the risk of infection and sepsis and should therefore be monitored carefully in anticancer treatments [83].

Recent investigations in other diseases have started to reveal how upregulation of TLRs in diseases is accomplished. CpG regulation of TLR2 is marked by unmethylation of the promoter in normal tissue and is probably not involved in tissue-specific regulation of TLR2 in normal cells. Furthermore, it was shown that $\mathrm{CpG}$ methylation of the proximal promoter may be involved in downregulation of TLR2 expression in tumors [85]. In addition, it was demonstrated that upregulation of TLR2 was associated with promoter hypomethylation [86]. A specific CpG region of the TLR2 promoter, adjacent to an SP1-binding site, was necessary and sufficient to maintain basal TLR2 promoter activity, suggesting that there are specific methylation patterns within the promoter [87]. This subsequently suggests that there may be oncogenic specific methylation patterns within the promoters of malignancies that regulate aberrant TLR expression, which could provide additional target markers for therapeutic options.

\section{Conclusions}

TLR-driven inflammation seems to play a role in furthering malignant development in a wide context of ways. It is crucial to understand not only the critical functions of how TLR signaling operates in tumor progression, but also the underlying epigenetic mechanisms that can be exploited for therapeutic options. In an ever-growing field, more research is required for therapeutic discoveries to add to our arsenal in order to combat cancerous phenotypes.

\section{Acknowledgements}

We thank Martin E. Fernandez-Zapico for critical reading of the manuscript and insightful comments. 


\section{References}

1 Gerttula S, Jin YS, Anderson KV: Zygotic expression and activity of the Drosophila Toll gene, a gene required maternally for embryonic dorsal-ventral pattern formation. Genetics 1988;119:123-133.

-2 Lemaitre B, Nicolas E, Michaut L, Reichhart JM, Hoffmann JA: The dorsoventral regulatory gene cassette spatzle/Toll/cactus controls the potent antifungal response in Drosophila adults. Cell 1996;86:973-983.

-3 Medzhitov R, Preston-Hurlburt P, Janeway CA Jr: A human homologue of the Drosophila Toll protein signals activation of adaptive immunity. Nature 1997;388:394-397.

4 Galluzzi L, et al: Trial Watch: experimental Toll-like receptor agonists for cancer therapy. Oncoimmunology 2012;1:699-716.

5 Kawai T, Akira S: The role of pattern-recognition receptors in innate immunity: update on Toll-like receptors. Nat Immunol 2010;11:373-384.

6 Basith S, Manavalan B, Yoo TH, Kim SG, Choi S: Roles of toll-like receptors in cancer: a double-edged sword for defense and offense. Arch Pharm Res 2012;35:1297-1316.

7 Hasan U, et al: Human TLR10 is a functional receptor, expressed by B cells and plasmacytoid dendritic cells, which activates gene transcription through MyD88. J Immunol 2005;174:2942-2950.

-8 Hansson GK, Edfeldt K: Toll to be paid at the gateway to the vessel wall. Arterioscler Thromb Vasc Biol 2005; 25:1085-1087.

-9 Huang B, et al: Toll-like receptors on tumor cells facilitate evasion of immune surveillance. Cancer Res 2005; 65:5009-5014

10 Huang B, Zhao J, Unkeless JC, Feng ZH, Xiong H: TLR signaling by tumor and immune cells: a double-edged sword. Oncogene 2008;27:218-224.

11 Oblak A, Jerala R: Toll-like receptor 4 activation in cancer progression and therapy. Clin Dev Immunol 2011; 2011:609579.

12 Rakoff-Nahoum S, Medzhitov R: Toll-like receptors and cancer. Nat Rev Cancer 2009;9:57-63.

13 Han X, et al: Epigenetic regulation of tumor necrosis factor alpha (TNFalpha) release in human macrophages by HIV-1 single-stranded RNA (ssRNA) is dependent on TLR8 signaling. J Biol Chem 2012;287:13778-13786.

14 Shi Z, et al: A novel Toll-like receptor that recognizes vesicular stomatitis virus. J Biol Chem 2011;286:45174524.

15 Lim KH, Staudt LM: Toll-like receptor signaling. Cold Spring Harb Perspect Biol 2013;5:a011247.

16 Kobayashi M, et al: Regulatory roles for MD-2 and TLR4 in ligand-induced receptor clustering. J Immunol 2006;176:6211-6218.

17 O'Neill LA, Bowie AG: The family of five: TIR-domain-containing adaptors in Toll-like receptor signalling. Nat Rev Immunol 2007;7:353-364.

18 Belinda LW, et al: SARM: a novel Toll-like receptor adaptor, is functionally conserved from arthropod to human. Mol Immunol 2008;45:1732-1742.

19 Carty M, et al: The human adaptor SARM negatively regulates adaptor protein TRIF-dependent Toll-like receptor signaling. Nat Immunol 2006; 7:1074-1081.

20 O'Neill LA: SIGIRR puts the brakes on Toll-like receptors. Nat Immunol 2003;4:823-824.

-21 Hoebe K, et al: Upregulation of costimulatory molecules induced by lipopolysaccharide and double-stranded RNA occurs by Trif-dependent and Trif-independent pathways. Nat Immunol 2003;4:1223-1229.

22 Banerjee S, et al: TLR signaling-mediated differential histone modification at IL-10 and IL-12 promoter region leads to functional impairments in tumor-associated macrophages. Carcinogenesis 2011;32:1789-1797.

23 Kagan JC, et al: TRAM couples endocytosis of Toll-like receptor 4 to the induction of interferon-beta. Nat Immunol 2008;9:361-368.

24 Sun R, et al: Toll-like receptor 3 (TLR3) induces apoptosis via death receptors and mitochondria by up-regulating the transactivating p63 isoform alpha (TAP63alpha). J Biol Chem 2011;286:15918-15928.

25 Aliprantis AO, Yang RB, Weiss DS, Godowski P, Zychlinsky A: The apoptotic signaling pathway activated by Toll-like receptor-2. EMBO J 2000;19:3325-3336.

26 Into T, et al: Stimulation of human Toll-like receptor (TLR) 2 and TLR6 with membrane lipoproteins of Mycoplasma fermentans induces apoptotic cell death after NF-kappa B activation. Cell Microbiol 2004;6:187-199.

27 Lopez M, et al: The 19-kDa Mycobacterium tuberculosis protein induces macrophage apoptosis through Tolllike receptor-2. J Immunol 2003;170:2409-2416.

-28 Oliveira RB, et al: Expression of Toll-like receptor 2 on human Schwann cells: a mechanism of nerve damage in leprosy. Infect Immun 2003;71:1427-1433.

29 Haase R, et al: A dominant role of Toll-like receptor 4 in the signaling of apoptosis in bacteria-faced macrophages. J Immunol 2003;171:4294-4303.

-30 Ruckdeschel K, et al: Signaling of apoptosis through TLRs critically involves toll/IL-1 receptor domaincontaining adapter inducing IFN-beta, but not MyD88, in bacteria-infected murine macrophages. J Immunol 2004;173:3320-3328.

-31 De Trez C, et al: TLR4 and Toll-IL-1 receptor domain-containing adapter-inducing IFN-beta, but not MyD88, regulate Escherichia coli-induced dendritic cell maturation and apoptosis in vivo. J Immunol 2005;175:839846.

32 Palaga T, et al: Notch signaling is activated by TLR stimulation and regulates macrophage functions. Eur J Immunol 2008;38:174-183. 
-33 Zhang Q, et al: Notch signal suppresses Toll-like receptor-triggered inflammatory responses in macrophages by inhibiting extracellular signal-regulated kinase 1/2-mediated nuclear factor $\mathrm{\kappa B}$ activation. J Biol Chem 2012;287:6208-6217.

34 Zeng Q, et al: Cross-talk between the Toll-like receptor 4 and Notch1 pathways augments the inflammatory response in the interstitial cells of stenotic human aortic valves. Circulation 2012;126:S222-S230.

-35 Bourke E, Bosisio D, Golay J, Polentarutti N, Mantovani A: The toll-like receptor repertoire of human B lymphocytes: inducible and selective expression of TLR9 and TLR10 in normal and transformed cells. Blood 2003; 102:956-963.

Grivennikov SI, Greten FR, Karin M: Immunity, inflammation, and cancer. Cell 2010;140:883-899. Bhattacharya D, Yusuf N: Expression of toll-like receptors on breast tumors: taking a toll on tumor microenvironment. Int J Breast Cancer 2012;2012:716564.

38 Gonzalez-Reyes S, et al: Study of TLR3, TLR4 and TLR9 in breast carcinomas and their association with metastasis. BMC Cancer 2010;10:665.

-39 Liao SJ, et al: Triggering of Toll-like receptor 4 on metastatic breast cancer cells promotes $\alpha v \beta 3$-mediated adhesion and invasive migration. Breast Cancer Res Treat 2012;133:853-863.

40 Ahmed A, Redmond HP, Wang JH: Links between Toll-like receptor 4 and breast cancer. Oncoimmunology 2013;2:e22945.

-41 Yang H, et al: Reduced expression of Toll-like receptor 4 inhibits human breast cancer cells proliferation and inflammatory cytokines secretion. J Exp Clin Cancer Res 2010;29:92.

42 Salaun B, Coste I, Rissoan MC, Lebecque SJ, Renno T: TLR3 can directly trigger apoptosis in human cancer cells. J Immunol 2006;176:4894-4901.

43 Amarante MK, et al: Toll-like receptor 3: implications for proinflammatory microenvironment in human breast cancer. Mol Biol Rep 2012;39:11087-11092.

44 Nomi N, Kodama S, Suzuki M: Toll-like receptor 3 signaling induces apoptosis in human head and neck cancer via survivin associated pathway. Oncol Rep 2010;24:225-231.

45 Matijevic T, Marjanovic M, Pavelic J: Functionally active toll-like receptor 3 on human primary and metastatic cancer cells. Scand J Immunol 2009;70:18-24.

46 Bernardo AR, Cosgaya JM, Aranda A, Jimenez-Lara AM: Synergy between RA and TLR3 promotes type I IFNdependent apoptosis through upregulation of TRAIL pathway in breast cancer cells. Cell Death Dis 2013; 4:e479.

47 Capece D, et al: The inflammatory microenvironment in hepatocellular carcinoma: a pivotal role for tumorassociated macrophages. Biomed Res Int 2013;2013:187204.

48 Liu C: Guardian and selective killer: the versatile functions of TLR3 in hepatocellular carcinoma. J Natl Cancer Inst 2012;104:1780-1782.

49 Jing YY, et al: Toll-like receptor 4 signaling promotes epithelial-mesenchymal transition in human hepatocellular carcinoma induced by lipopolysaccharide. BMC Med 2012;10:98.

50 Liang B, et al: Myeloid differentiation factor 88 promotes growth and metastasis of human hepatocellular carcinoma. Clin Cancer Res 2013;19:2905-2916.

51 Ochi A, et al: Toll-like receptor 7 regulates pancreatic carcinogenesis in mice and humans. J Clin Invest 2012; 122:4118-4129.

52 Huynh AS, et al: Novel toll-like receptor 2 ligands for targeted pancreatic cancer imaging and immunotherapy. J Med Chem 2012;55:9751-9762.

53 Zambirinis CP, Miller G: Signaling via MYD88 in the pancreatic tumor microenvironment: a double-edged sword. Oncoimmunology 2013;2:e22567.

54 Morse DL, et al: Identification of novel pancreatic adenocarcinoma cell-surface targets by gene expression profiling and tissue microarray. Biochem Pharmacol 2010;80:748-754.

55 Ochi A, et al: MyD88 inhibition amplifies dendritic cell capacity to promote pancreatic carcinogenesis via Th2 cells. J Exp Med 2012;209:1671-1687.

56 Pinto A, Morello S, Sorrentino R: Lung cancer and Toll-like receptors. Cancer Immunol Immunother 2011;60: 1211-1220.

57 Cherfils-Vicini J, et al: Triggering of TLR7 and TLR8 expressed by human lung cancer cells induces cell survival and chemoresistance. J Clin Invest 2010;120:1285-1297.

58 Zhang YB, et al: Increased expression of Toll-like receptors 4 and 9 in human lung cancer. Mol Biol Rep 2009; $36: 1475-1481$

59 Ren T, et al: Functional expression of TLR9 is associated to the metastatic potential of human lung cancer cell: functional active role of TLR9 on tumor metastasis. Cancer Biol Ther 2007;6:1704-1709.

60 Ren T, et al: TLR9 signaling promotes tumor progression of human lung cancer cell in vivo. Pathol Oncol Res 2009;15:623-630.

61 Schmidt M, et al: Crucial role for human Toll-like receptor 4 in the development of contact allergy to nickel. Nat Immunol 2010;11:814-819.

62 Xu Z, Ren T, Xiao C, Li H, Wu T: Nickel promotes the invasive potential of human lung cancer cells via TLR4/ MyD88 signaling. Toxicology 2011;285:25-30.

63 Ngo VN, et al: Oncogenically active MYD88 mutations in human lymphoma. Nature 2011;470:115-119.

64 Jeelall YS, Horikawa K: Oncogenic MYD88 mutation drives Toll pathway to lymphoma. Immunol Cell Biol 2011 ; 89:659-660. 
Gachard N, et al: IGHV gene features and MYD88 L265P mutation separate the three marginal zone lymphoma entities and Waldenström macroglobulinemia/lymphoplasmacytic lymphomas. Leukemia 2013;27:183-189.

66 Treon SP, et al: MYD88 L265P somatic mutation in Waldenström's macroglobulinemia. N Engl J Med 2012; 367:826-833.

67 Wang L, et al: Toll-like receptor-4 signaling in mantle cell lymphoma: effects on tumor growth and immune evasion. Cancer 2013;119:782-791.

68 Xu Y, et al: Expression and function of toll-like receptors in multiple myeloma patients: toll-like receptor ligands promote multiple myeloma cell growth and survival via activation of nuclear factor- $\mathrm{kB}$. Br J Haematol 2010;150:543-553.

69 Bell JK: The TOLL of inflammation in multiple myeloma. Cancer Biol Ther 2011;11:68-70.

70 Bao H, et al: Triggering of toll-like receptor-4 in human multiple myeloma cells promotes proliferation and alters cell responses to immune and chemotherapy drug attack. Cancer Biol Ther 2011;11:58-67.

71 Butler JS, Dent SY: The role of chromatin modifiers in normal and malignant hematopoiesis. Blood 2013;121: 3076-3084.

72 Jenuwein T, Allis CD: Translating the histone code. Science 2001;293:1074-1080.

73 Li B, Carey M, Workman JL: The role of chromatin during transcription. Cell 2007;128:707-719.

74 Foster SL, Medzhitov R: Gene-specific control of the TLR-induced inflammatory response. Clin Immunol 2009; 130:7-15.

75 Hake SB, Xiao A, Allis CD: Linking the epigenetic 'language' of covalent histone modifications to cancer. Br J Cancer 2004;90:761-769.

76 Janson PC, Winqvist 0: Epigenetics - the key to understand immune responses in health and disease. Am J Reprod Immunol 2011;66(suppl 1):72-74.

77 Berger SL: The complex language of chromatin regulation during transcription. Nature 2007;447:407-412.

78 Bierne H, Hamon M, Cossart P: Epigenetics and bacterial infections. Cold Spring Harb Perspect Med 2012; 2:a010272.

79 Hideshima T, Bergsagel PL, Kuehl WM, Anderson KC: Advances in biology of multiple myeloma: clinical applications. Blood 2004;104:607-618.

80 van Horssen R, Ten Hagen TL, Eggermont AM: TNF-alpha in cancer treatment: molecular insights, antitumor effects, and clinical utility. Oncologist 2006;11:397-408.

81 Sullivan KE, et al: Epigenetic regulation of tumor necrosis factor alpha. Mol Cell Biol 2007;27:5147-5160.

-82 Zauner L, et al: TLR9 triggering in Burkitt's lymphoma cell lines suppresses the EBV BZLF1 transcription via histone modification. Oncogene 2010;29:4588-4598.

-83 Roger T, et al: Histone deacetylase inhibitors impair innate immune responses to Toll-like receptor agonists and to infection. Blood 2011;117:1205-1217.

84 Song W, et al: HDAC inhibition by LBH589 affects the phenotype and function of human myeloid dendritic cells. Leukemia 2011;25:161-168.

85 Haehnel V, Schwarzfischer L, Fenton MJ, Rehli M: Transcriptional regulation of the human toll-like receptor 2 gene in monocytes and macrophages. J Immunol 2002;168:5629-5637.

-86 Shuto T, et al: Promoter hypomethylation of Toll-like receptor-2 gene is associated with increased proinflammatory response toward bacterial peptidoglycan in cystic fibrosis bronchial epithelial cells. FASEB J 2006;20: 782-784.

-87 Furuta T, et al: DNA demethylation-dependent enhancement of toll-like receptor-2 gene expression in cystic fibrosis epithelial cells involves SP1-activated transcription. BMC Mol Biol 2008;9:39. 